\title{
El Consejo de Derechos Humanos en el nuevo escenario mundial y los nuevos mecanismos de revisión ${ }^{*}$
}

\author{
Irene Acevedo Albornoz \\ Ministerio de Relaciones Exteriores, Santiago, Chile. \\ Email: irene.acevedo@usach.cl \\ Jorge Riquelme Rivera \\ Universidad de Chile, Santiago, Chile. \\ Email: jlriquel@uc.cl
}

\begin{abstract}
Resumen: El trabajo analiza el tema de los derechos humanos bajo la perspectiva de la política multilateral. En esta línea, se concentra en las innovaciones que ha implicado el establecimiento del Consejo de Derechos Humanos, en lugar de la antigua Comisión de Derechos Humanos. Para tal efecto, se pone un énfasis especial en el estudio de las discusiones políticas que se dieron en el seno de la Comunidad Internacional, durante el proceso de constitución del nuevo Consejo, así como en los nuevos mecanismos de revisión que éste contempla.

Palabras claves: Multilateralismo, Naciones Unidas, Comisión de Derechos Humanos, Consejo de Derechos Humanos
\end{abstract}

\section{The Human Rights Council in the new international order and the new mechanisms of revision}

\begin{abstract}
This paper analyzes the Human Rights topic under a multilateral policy perspective. In this regard, it focuses on the innovations that have entailed the establishment of the Human Rights Council, instead of the former Human Rights Commission. For that purpose, special emphasis is placed on the study of political discussions within the International Community, during the process of establishment of the new Council, as well as the new review mechanisms that it contemplates.

Key Words: Multilateralism, United Nations, Human Rights Commission, Human Rights Council
\end{abstract}

\section{O Conselho de Direitos Humanos no novo ordem interna- cional e os novos mecanismos para revisão}

Resumo: Este artigo analisa a questão dos direitos humanos sob a perspectiva da política multilateral. Nesta linha, centra-se em inovações que envolveu o estabelecimento do Conselho de Direitos Humanos, em vez da antiga Comissão de Direitos Humanos. Para este fim, ênfase especial é colocada no estudo das discussões políticas que tiveram lugar no seio da comunidade internacional durante o processo 
de criação do novo Conselho, bem como examinar as novas disposições que contempla.

Palavras-chave: Multilateralismo, Comissão das Nações Unidas sobre Direitos Humanos, Conselho de Direitos Humanos

\section{Introducción}

En el contexto de la política internacional, el multilateralismo contemporáneo tiene su origen en la Sociedad de Naciones, que tras la Primera Guerra Mundial se conformó con el propósito esencial de promover un escenario internacional estable y pacífico. El fracaso de esta organización y la consecuente conflagración mundial que se desarrolló entre 1939 y 1945 decantaron en una nueva institución, la Organización de las Naciones Unidas, núcleo del multilateralismo actual y foro principal del diálogo político de la comunidad internacional.

La política multilateral promueve la existencia de reglas claras y disciplinas transparentes, avaladas por un sistema internacional que otorga oportunidades a todos los actores. Es decir, el multilateralismo es la herramienta primordial para los países de menor tamaño relativo para incidir en los sucesos de la política mundial, más allá de la mera política del balance de poder. En el mundo actual, un multilateralismo que promueva el respeto de la pluralidad de visiones, es una pieza fundamental para enfrentar los desafíos del mundo global.

Bajo este marco general, los derechos humanos han tenido una relevante evolución conceptual y teórica, así como un importante desarrollo como instrumento de política exterior, particularmente en los países occidentales. Los derechos humanos hoy son un elemento central del debate en Naciones Unidas que, junto a la superación de la pobreza, el cambio climático y la equidad de género, se han convertido en uno de los temas transversales, que están presentes en prácticamente todas las iniciativas que se desarrollan en el ámbito de la política multilateral.

Según sostiene el profesor José Zalaquett (2010: 12), luego de la Segunda Guerra Mundial se produjo una internacionalización sistemática de los derechos humanos, comenzando por la Declaración Universal de los Derechos Humanos de Naciones Unidas, del año 1948. Luego de esta Declaración se adoptó en la Organización, en 1966 el Pacto Internacional de Derechos Civiles y Políticos y el Pacto Internacional de Derechos Económicos, Sociales y Culturales, los cuales entraron en vigor en 1976. De manera paralela a estas instancias, se han acordado numerosos otros pactos o convenciones sobre esta materia en Naciones Unidas y en organizaciones regionales, como los instrumentos de derechos humanos en el seno de la Organización de Estados Americanos (OEA). 
Respecto de la Comisión de Derechos Humanos, ésta fue creada en 1946 por el Consejo Económico y Social (ECOSOC), en cumplimiento del Art. 68 de la Carta de Naciones Unidas y estuvo conformada en un principio, por 18 Estados que luego aumentaron a 53 de acuerdo al sistema de rotación geográfica. La Comisión centró sus esfuerzos en la creación de normas internacionales sobre derechos humanos, para lo cual se estableció un grupo de redacción presidido por Eleonor Roosevelt, quien tuvo la misión de redactar un documento consensuado sobre esta temática. De esta labor surgió en 1948, la Declaración Universal de los Derechos Humanos.

La Comisión de Derechos Humanos fue el foro más importante del mundo en dicha materia desde su conformación en 1946. La Comisión fue establecida para proteger los derechos y libertades fundamentales de todos, sin hacer distinciones por motivos de raza, sexo, idioma o religión (Art. 1 de la Carta de Naciones Unidas), ampliando sus niveles de trabajo en función de la complejidad del mundo y la necesidad de ajustar su mandato a los requerimientos de la dinámica política internacional.

Sin perjuicio de lo anterior, en los albores del siglo XXI se hacía evidente la necesidad de incorporar cambios en la institucionalidad que la comunidad de naciones contemplaba para el ámbito de los derechos humanos, en tanto el escenario internacional se había crecientemente complejizado desde fines de la década de los ochenta. Con el fin de la Guerra Fría, el derrumbe del bloque soviético y el consecuente fin de los socialismos reales, Naciones Unidas debió enfrentar un abanico de nuevos problemas que el dinámico proceso de globalización ponía en evidencia.

El académico chileno José Morandé plantea que, a la preocupación tradicional de los Estados respecto de la guerra y la paz, la expansión hegemónica y los nacionalismos, se sumaron entonces las armas de destrucción masiva, la destrucción progresiva del medio ambiente, la violación sistemática de los derechos humanos, la expansión y gravitación de las religiones y los peligros de un terrorismo transnacional de gran impacto en la política mundial (Morandé, 2004)

Lo anterior aconteció en un escenario internacional marcado por una brusca transformación del balance de fuerzas de la era bipolar, que se tradujo en una situación transitoriamente unipolar; un aumento en el número y variedad de los actores internacionales y transnacionales; la pérdida de relevancia del Estado nación como protagonista central del sistema internacional; y los diversos procesos de integración regional que se ensayaban en el mundo. A estos complejos desafíos se sumaron en septiembre de 2001 los atentados a las Torres Gemelas en Nueva York y al Pentágono en Washington. Desde entonces, nuevos atentados se han perpetrado en distintas zonas del orbe, los que se unen a una serie de escenarios de conflictos e inestabilidad sin una perspectiva clara de resolución.

En este complejo escenario global, el año 2005 ya era patente la necesidad de llegar a un consenso en la comunidad internacional sobre la 
creación de una renovada entidad que respondiera a las nuevas demandas de los Estados, que requerían de un ente con mayores atribuciones en el ámbito de los derechos humanos. De este modo, en el año 2006 se creó el Consejo de Derechos Humanos, mediante la resolución A/RES/60/251 de la Asamblea General. El Consejo es un órgano intergubernamental compuesto por 47 Estados responsables de la promoción y protección de los derechos humanos en el mundo. Tras celebrar su primera sesión en 2007, el Consejo elaboró un paquete de construcción institucional que guiaría su trabajo en el futuro.

Como se abundará más adelante, entre los elementos innovadores del Consejo, se cuenta la creación del mecanismo de Examen Periódico Universal (EPU), a través del cual se examina la situación de los derechos humanos de todos los Estados miembros de Naciones Unidas. También destaca la creación de un Comité Asesor, que se ocupa de diversas cuestiones temáticas; así como el establecimiento de un nuevo mecanismo de Método de Denuncias, que permite que individuos y organizaciones presenten acusaciones sobre violaciones de derechos humanos, con el fin de ser examinadas por el Consejo.

Cabe considerar que entre los propósitos y principios de la Carta de Naciones Unidas, se deja expresa constancia de la necesidad de estimular el respeto de los derechos humanos y de las libertades fundamentales de todos sin distinción. En su Art. 13 se establece que la Asamblea General, principal órgano de Naciones Unidas, promoverá el estudio y hará recomendaciones con el fin de fomentar y hacer efectivos los derechos y libertades fundamentales. Con el propósito de crear las condiciones de estabilidad y bienestar necesarias para las relaciones pacíficas y amistosas entre las naciones, el Art. 55 letra c) sostiene que la organización promoverá el respeto universal de los derechos humanos y de las libertades, ordenando que el ECOSOC haga recomendaciones con el objeto de promover el respeto de los derechos y la efectividad de tales derechos y libertades (Art. 62).

A su vez, el Art. 76 reconoce la interdependencia de los pueblos del mundo en la promoción de los derechos humanos. De este modo, la Carta de San Francisco, que dio origen al sistema de Naciones Unidas, contempló entre sus temas prioritarios la promoción y la protección de los derechos humanos como una condición básica y necesaria del bienestar humano.

\section{El proceso de negociación en el sistema de Naciones Unidas y sus órganos subsidiarios}

La negociación internacional se realiza entre Estados caracterizados por las asimetrías y diferencias en sus respectivos pesos específicos. El mundo global está compuesto por países que presentan distintos niveles de desarrollo económico, visiones culturales, capacidades tecnológicas, esti- 
los de diálogo, población y territorio, entre otros. Por esta razón, la negociación multilateral presenta aditamentos que le son propios, como son la formación de alianzas o coaliciones en temas específicos, que pretenden aunar fuerzas entre los países en torno a objetivos comunes.

Las negociaciones son afectadas por cuestiones culturales, sean éstas de los mismos negociadores o propios de las partes a las cuales representan. En ocasiones, la negociación multilateral pone en juego una pluralidad de temas conectados entre sí, que pueden ser objeto de una o varias sesiones simultáneas o paralelas. Siendo el concepto de derechos humanos polisémico y, por ende, generador de múltiples controversias, las aspiraciones, intereses y disímiles formas de ver y construir el mundo hacen de la política internacional en esta materia una fuente inagotable de problemáticas por resolver.

La Carta de Naciones Unidas establece un principio a partir del cual se han orientado los acuerdos, ya sean globales o regionales. Según éste, los miembros de la Organización deben arreglar sus controversias internacionales utilizando medios pacíficos, de tal manera que no se ponga en peligro la paz y la seguridad internacionales, ni la justicia entre las naciones (Art. 2.3 de la Carta de Naciones Unidas). Para ello, la Asamblea General de Naciones Unidas aprobó en 1998 la resolución A/RES/53/ 101, que contiene los Principios y Directrices para las Negociaciones Internacionales ${ }^{1}$.

En este marco general, si bien el consenso es un mecanismo que se ha adoptado para resolver la mayoría de las cuestiones que hoy son debatidas en Naciones Unidas y sus órganos subsidiarios, el sistema de votaciones ha permanecido inalterable en el tema de los derechos humanos, tanto en la antigua Comisión de Derechos Humanos como en el actual Consejo. Lo anterior, en razón de que el entendimiento negociado que busca consensuar posiciones no ha sido posible de instalar en esta entidad, a pesar de las muchas ventajas que posee, tales como la estabilidad, adherencia y el posterior cumplimiento de sus disposiciones. El Consejo de Derechos Humanos continúa usando el sistema de las mayorías que lo mantiene con una cierta debilidad ante los acuerdos que alcanza. Asimismo, las resoluciones que condenan violaciones a los derechos humanos continúan siendo recibidas por los Estados a nivel de sugerencias o, en el mejor de los casos, como simples recomendaciones.

Una manera de superar algunas trabas de la negociación es recurrir a una negociación oficiosa, que se realiza mediante el uso de conversaciones de pasillo que se llevan a cabo entre una o más delegaciones, con el fin de sincerar posiciones y solicitar flexibilidad respecto de algún punto en que el Estado no puede manifestarse oficialmente ${ }^{2}$. En el contexto de estas negociaciones, se hace patente que la política exterior de los Estados no sólo es el reflejo de los intereses nacionales, sino que también responde a presiones nacionales e internacionales de grupos ideológicos que ejercen una considerable coacción en la toma de decisiones. 


\section{Naciones Unidas y la Comisión de Derechos Humanos}

La dinámica de la política internacional, en la cual los Estados han basado su accionar en el diálogo y el refuerzo del multilateralismo, ha permitido proteger y validar el comportamiento de los países de menor desarrollo, no sólo en las materias tocantes al progreso económico, sino también en temas relacionados con el bienestar y la seguridad humana. En tal sentido, uno de los conceptos que más adquirió relevancia en el ámbito multilateral en el último decenio del siglo XX fue el de la cooperación. En esta esfera, los Estados en el mundo globalizado se fueron identificando positivamente entre sí y percibieron, a la luz de los acontecimientos, que la seguridad y el progreso de cada uno era responsabilidad de todos.

De este modo, Naciones Unidas se ha ido consolidando a través del acuerdo de los Estados partes. La creación de entidades al interior de este sistema ha desarrollado un marco institucional en el cual operan ciertos estándares de comportamiento, la aceptación de reglas y el cumplimiento de los compromisos acordados, los que a pesar que experimenten una cierta fragilidad, igualmente son condicionados por mecanismos de carácter coercitivo (aislamiento, sanciones económicas o retiro de apoyos a candidaturas, entre otros), que permiten fijar ciertas normas de comportamiento. ${ }^{3}$ Ello permite vislumbrar un escenario internacional que sería esencialmente anárquico, pero donde, no obstante, se observa una notable tendencia a institucionalizar las relaciones entre los países mediante el derecho internacional.

En este marco, y considerando los supuestos del análisis institucional (North, 1990), es posible apreciar que, en el presente, el sistema de Naciones Unidas -núcleo de la política multilateral en el mundo- ha llegado a convertirse en una verdadera institución en términos de las creencias, normas y reglas que le han permitido el desarrollo de la estructura y funciones para las cuales fue creada. Las instituciones van consolidando su fuerza con el paso del tiempo y por ello es que, a pesar de que los Estados miembros presentan diferencias irreconciliables en torno al tema de los derechos humanos, aquellos permanecerán subsumidos al interior de la Organización.

En lo referido a la Comisión de Derechos Humanos en particular, es posible observar que con el devenir del tiempo y tras cumplir medio siglo desde su creación, aquella venía experimentado una inusual paradoja en su existencia, por cuanto a medida que se incrementaba su prestigio simbólico, por otra parte iba perdiendo eficacia práctica. Las reiteradas resoluciones de condena a algunos Estados que presentaban claras e incuestionables violaciones a los derechos humanos, se fueron convirtiendo en documentos que sólo agregaban números y años a frases previamente acordadas, asegurando así su rápida adopción, pero afectando su utilidad y eficacia concreta. Asimismo, el proceso de discusión y debate previo a la votación de las resoluciones, se había convertido en un ritual anual con escasas posibilidades de desarrollar los contenidos o avanzar en torno a los problemas puntuales que éstas abordaban. De tal manera, la Comisión de Derechos Hu- 
manos fue experimentando una sostenida tendencia a la inercia.

Sin embargo, la reflexión que se desarrolló en los albores de este siglo, en el marco de la reforma de Naciones Unidas, posibilitó que a través de largos procesos la estructura e institucionalidad de la Organización redefiniera la identidad e intereses de la Comisión. En tal sentido, debido a la relevancia creciente del problema global en torno a la definición de los derechos humanos, se requirió que la temática fuera vista como un problema a resolver en forma conjunta por los Estados, tomando en cuenta las diferencias existentes entre éstos, respecto de la responsabilidad, los efectos adversos y las capacidades de afrontar estas materias.

\section{El surgimiento del Consejo de Derechos Humanos}

En 1948 surgió la Declaración Universal de los Derechos Humanos. Al no lograrse un consenso sobre ésta, la Declaración fue adoptada en la Asamblea General por votación (se obtuvieron 48 votos a favor, cero en contra y 8 abstenciones). No obstante, no hubo acuerdo para hacer de esta Declaración una convención jurídicamente obligatoria (Figueroa, 2010: 252).

Dicha Declaración no tiene un estatus jurídico vinculante, sino ostenta el valor que se le asigna a una resolución, es decir, el de una recomendación para los países miembros de Naciones Unidas. No obstante lo anterior, una resolución contribuye en parte, a la creación de una costumbre de derecho internacional, que posteriormente constituye una fuente del derecho. Sin embargo, su fuerza intrínseca radica en la naturaleza del tema que trata y la amplia difusión que el instrumento ha tenido en el mundo desde su aprobación, llegando a valorarse como el manifiesto donde están contenidos implícitamente todos los derechos humanos. Sin perjuicio de lo anterior, el universo de tales derechos no fue abarcado de manera concreta en el texto del documento, razón por la cual se generó la necesidad de elaborar una serie de instrumentos internacionales, que han desarrollado diversas áreas de la vida humana y que no fueron contemplados originalmente.

De esta manera, a partir de la década del sesenta surgieron convenciones internacionales respecto a diversos temas, tales como la discriminación racial, la discriminación contra la mujer, la tortura, los derechos del niño, la protección de los trabajadores migrantes, y en este nuevo siglo, sobre las desapariciones forzadas y las personas con discapacidad. Todavía se debate en el terreno multilateral lo tocante al derecho al desarrollo, la alimentación y la paz. Bajo el paraguas de la Declaración Universal de los Derechos Humanos, se negociaron diversos instrumentos en la materia, como el Pacto de Derechos Civiles y Políticos y el Pacto Internacional sobre Derechos Económicos, Sociales y Culturales, aprobados por la Asamblea General en 1966.

En este marco, la antigua Comisión de Derechos Humanos otorgaba orientaciones generales, estudiaba problemas de derechos humanos, desa- 
rrollaba y codificaba nuevas normas internacionales y vigilaba la observancia de los derechos humanos en todo el mundo. En su calidad de principal órgano intergubernamental de formulación de políticas en el ámbito de los derechos humanos de Naciones Unidas, la Comisión estaba facultada para examinar la situación de los derechos humanos en cualquier parte del mundo, así como la información proveniente de los Estados, las Organizaciones No Gubernamentales (ONGs) y otras fuentes de relevancia (Naciones Unidas, 2000: 256).

Con el fin de avanzar hacia mayores estadios de consolidación de los conceptos de promoción y protección de los derechos humanos, se establecieron mecanismos para hacer efectiva su vigencia. Para este efecto se conformaron grupos de expertos y relatores especiales, llegando estos últimos a constituirse en figuras relevante en aquellos lugares donde se necesitaba salvaguardar los derechos de los grupos más vulnerables de la sociedad. Así surge el concepto de salvaguardia, que el actual Consejo de Derechos Humanos ha asumido como fundamental, legislando sobre este mecanismo el año 2007, cuando se dictó el Código de Conducta para un mejor desempeño de esta labor.

Ante los precarios resultados de la Comisión de Derechos Humanos y las numerosas críticas, debido a su escasa operatividad y supuesta politización, y con el fin de fortalecer el sistema de protección internacional de los derechos humanos, desde el seno de Naciones Unidas se planteó la necesidad de suprimir la Comisión y sustituirla por un Consejo de Derechos Humanos, mejor organizado y más efectivo.

Los orígenes de este cambio hay que buscarlos en el proceso de reforma de Naciones Unidas, impulsado por el ex Secretario General, Kofi Annan, quien quiso otorgar una mayor importancia al tema de los derechos humanos en la actividad de la Organización.

Dos son los documentos inspiradores de este proceso: el Informe de Expertos de alto nivel sobre Amenazas, Desafíos y el Cambio, de 2003, y el Informe de 2005 In Larger Freedom. En ambos escritos se plantea la necesidad de continuar el trabajo de definición de estándares, empezado por la Comisión en el campo de los derechos humanos, así como el fortalecimiento de los mecanismos para su monitoreo y protección. En la primera década de este siglo, la Comisión de Derechos Humanos había llegado a un punto de quiebre. A pesar del trabajo realizado, la instancia se había vuelto un mecanismo insuficiente para garantizar el respeto de los derechos humanos en el mundo.

Frente a las críticas, en su informe de 2005 el entonces Secretario General Annan avanzó en la propuesta de reemplazar la Comisión con un nuevo Consejo, cuyo estatus y funcionamiento lo tornarían más fuerte y eficiente que su predecesora. La propuesta fue acogida por los Jefes de Estado y de Gobierno de los países miembros de Naciones Unidas en la Cumbre Mundial de 2005, donde se planteó la necesidad de fortalecer los 
derechos humanos como el tercer pilar de la Organización, junto a los temas de Paz y Seguridad internacionales y Desarrollo.

De este modo, se aprobó el 16 de septiembre de 2005 la creación de un Consejo de Derechos Humanos, responsable de promover el respeto universal de la protección de todos los derechos humanos y libertades fundamentales, estudiar situaciones de violaciones graves y sistemáticas de los derechos humanos y promover la coordinación eficaz y la incorporación de los derechos humanos en las actividades de todo el sistema de Naciones Unidas. De esta manera, el 15 de marzo del año 2006, la Asamblea General de Naciones Unidas aprobó la creación del Consejo de Derechos Humanos, mediante la resolución A/RES/60/251.

Entre las novedades del Consejo, se cuenta el que los miembros del nuevo Consejo de Derechos Humanos son elegidos por una mayoría de 96 votos a favor, los que sirven por un período de tres años, sin poder ser reelegidos inmediatamente después de haber ocupado el puesto por dos períodos consecutivos. Los Estados deben comprometerse en mantener los máximos estándares de respeto de los derechos humanos y a someterse a revisiones periódicas por expertos de Naciones Unidas. En concreto, el Consejo es actualmente el principal foro la Organización para el diálogo y la cooperación en materia de derechos humanos. Se concentra en ayudar a los Estados miembros a cumplir con sus obligaciones por medio del diálogo, el desarrollo de capacidades y asistencia técnica. También puede hacer recomendaciones a la Asamblea General, con el fin de impulsar un mayor desarrollo del derecho internacional en el ámbito de los derechos humanos.

Durante las negociaciones de la resolución que creó el Consejo de Derechos Humanos, algunos países manifestaron sus aprensiones respecto del texto aprobado, en razón que no reflejaba adecuadamente sus intereses nacionales. El proyecto de resolución fue adoptado por 170 votos a favor, cuatro en contra (los de Estados Unidos, Israel, Palau y las Islas Marshall) y tres abstenciones (Irán, Belarus y Venezuela). Al explicar su voto en contra, el representante de Estados Unidos, Embajador John Bolton, expresó su escepticismo sobre el futuro del Consejo, pero manifestó el compromiso de este país a cooperar con otros Estados miembros para fortalecerlo y colaborar en su funcionamiento. Cuba por otro lado, criticó el texto de la resolución, pero lo votó a favor, mientras que Venezuela expresó distintas objeciones, pero no la votó en contra.

Antes de la votación Cuba hizo una declaración, planteando que el país votaría a favor de la resolución a pesar de que el texto final reflejaba los intereses de las potencias tradicionales, las que a su juicio se empeñaban en que siguiera prevaleciendo el enfoque punitivo y sancionador en esta nueva entidad.

Por su parte, Venezuela declaró antes de la votación que su posición era estar en contra, sin embargo se abstendrían, con el fin de no compartir la posición con Estados Unidos. ${ }^{4}$ Asimismo, Venezuela manifestó una cla- 
ra aprensión sobre la actuación que tendrían las ONGs, detalladas en el párrafo decimotercero del texto. Esta posición era contraria a la sostenida por la Unión Europea, Islandia y Chile, quienes han mantenido la posición de acoger con beneplácito la incorporación del trabajo de las ONGs en la labor del Consejo, otorgándole una especial relevancia a su contribución en la promoción de los derechos humanos.

Una vez sometido el texto a votación, Estados Unidos declaró que su decisión de votar en contra obedecía a que no confiaban en que el texto asegurara que el Consejo de Derechos Humanos sería mejor que su predecesora. Sin embargo, apoyaría el esfuerzo por fortalecer el Consejo, esperando que se examinara con seriedad su estructura y su labor. Para los Estados Unidos el Consejo no debía dar lugar a países donde existieran pruebas objetivas de violaciones flagrantes y sistemáticas de los derechos humanos, o en que se hayan aplicado sanciones de las Naciones Unidas debido a violaciones de estos derechos. Con el fin de sustentar una posición con mayor fundamento, el delegado norteamericano citó las palabras del Secretario General de Naciones Unidas Annan, quien sostuvo respecto del problema fundamental de la antigua Comisión, que en el pasado hubo Estados que se habían hecho miembros de la Comisión no para afianzar los derechos humanos, sino para protegerse contra las críticas o para criticar a otros.

Respecto del alcance que hiciera Estados Unidos sobre abstenerse de votar por un Estado candidato que haya sido sancionado por el Consejo de Seguridad por motivos vinculados a los derechos humanos, la Unión Europea, los países de la CANZ (Canadá, Australia y Nueva Zelandia), Japón, y otros Estados se comprometieron a mantener dicho lineamiento. En una posición contrapuesta declararon Sudán, India e Irán, que visualizaban en la relación entre el Consejo de Derechos Humanos y el Consejo de Seguridad una injerencia peligrosa de éste último, ante lo cual la Asamblea General debía tener la última palabra.

Por otra parte, los países miembros de la organización de la Conferencia Islámica manifestaron que distaban mucho de sentirse satisfechos con esta resolución, la que debería haber tenido referencias inequívocas, tanto en el preámbulo como en la parte dispositiva, respecto de los actos de incitación, odio e intolerancia religiosa. Esta declaración, efectuada por Yemen en nombre del grupo, expresaba el temor de estos países a que bajo el paraguas del principio de la libertad de expresión, los países musulmanes se sintieran menoscabados en sus creencias y particularidades culturales. Al mismo tiempo, los países de la Conferencia Islámica, junto a miembros del CARICOM (Caribean Community), China, Federación Rusa, Suiza e Israel -entre otros-, manifestaron la necesidad de evitar la politización, la selectividad y los dobles raseros del Consejo, actitudes que habían sido la característica principal de la Comisión, respecto de la adopción de resoluciones condenatorias a Estados que, por lo general, pertenecían al mundo en desarrollo.

Dentro de los temas que los países desarrollados lamentaron que no 
haya habido acuerdo, fue el estatus del Consejo, que no pudo ser elevado a la categoría de órgano Principal de Naciones Unidas y que los miembros fueran elegidos por una mayoría calificada de dos tercios. Por otra parte, la frustración mayor de los países en desarrollo fue que la entidad no pusiera más énfasis en un programa enfocado al desarrollo, tema que aún no puede ser consensuado, en particular respecto de la adopción de una resolución que establezca la existencia del derecho al desarrollo.

Las declaraciones efectuadas con ocasión de la votación de la resolución que le dio vida al Consejo, se orientaron a manifestar problemas específicos con los que conviven ciertos Estados. Las frustraciones de países desarrollados y en desarrollo se refieren a la distancia de ambos conglomerados con el punto medio que finalmente resultó de las negociaciones y que dejó con cierto descontento a muchos países. Sin embargo, el proceso constituyó un reflejo del esfuerzo con que los Estados se sentaron a dialogar y a buscar puntos de encuentro. El presidente de la Asamblea General en 2006, el sueco Jan Eliasson, terminó la jornada de votación, expresando que los Estados se deben responsabilizar de las actividades en la esfera de los derechos humanos, más allá de cuestionar si el tema tiene aún los ribetes de las constantes y tradicionales disputas entre los países del Norte y el Sur. En tal sentido, la resolución dejó establecido que la cooperación y el diálogo debían guiar los trabajos del Consejo, con el fin de lograr el progreso esperado.

\section{Innovaciones introducidas con el nuevo Consejo de Derechos Humanos y sus consecuencias}

La sustitución de la antigua Comisión de Derechos Humanos por el Consejo implicó cambios significativos en la estructura y funcionamiento del nuevo organismo, con el fin principal de responder a las acusaciones de politización de las que había sido objeto. Este nuevo organismo sería, de conformidad con el Art. 22 de la Carta, un órgano subsidiario de la Asamblea General y, por lo tanto, de una mayor jerarquía que la Comisión. No obstante, a este respecto el profesor Carlos Villán Durán lamenta que el Consejo no pueda informar igualmente de manera directa al Consejo de Seguridad, por cuanto existe una estrecha relación entre las violaciones masivas de los derechos humanos y el mantenimiento de la paz y seguridad internacionales (Villán Durán, 2006: 2).

El Consejo sesiona un mínimo de tres veces por año, incluido un período de sesiones principal, con una duración total no inferior a diez semanas, ${ }^{5}$ lo que significa un cambio con respecto a la Comisión, convirtiéndolo en un órgano casi permanente. Estos hechos contribuyen a consolidar el énfasis en los derechos humanos promovido con la reestructuración de las Naciones Unidas. Asimismo, está compuesto por 47 Estados, considerando una representación geográfica equitativa. Los miembros son elegidos por períodos de tres años en votación secreta, por la mayoría de los 
miembros de la Asamblea General, no pudiendo ningún Estado miembro ser reelegido inmediatamente luego de dos períodos consecutivos.

Un asunto relevante que cabe subrayar, es que la distribución de los Estados Miembros en el Consejo facilita una efectiva rotación de todos ellos y evita que algunos Estados actúen de facto de manera permanente. En el pasado, China y Estados Unidos habían actuado como actores preponderantes, fiscalizando el respeto de los derechos humanos en otros Estados. El nuevo Consejo, al contemplar la participación de todos los países $\mathrm{y}$ al responder directamente a la Asamblea General, limita la posible ingerencia de poder por parte de los países más poderosos.

En la creación del Consejo de Derechos Humanos, y ante las críticas sobre la excesiva politización de la antigua Comisión, se introducen tres correctivos. Primero, al elegir a los miembros del Consejo los Estados deben tener en cuenta la contribución de los candidatos a la promoción y protección de los derechos humanos y las promesas y contribuciones voluntarias que hayan hecho al respecto. Segundo, se prevé que la Asamblea General podrá suspender por mayoría de dos tercios a todo miembro del Consejo que cometa violaciones graves y sistemáticas de los derechos humanos. Y tercero, los miembros del Consejo deberán defender las más altas exigencias en la promoción y protección de los derechos humanos, cooperar plenamente con el Consejo y ser examinados con arreglo al mecanismo del EPU durante su periodo como miembro.

Pese a lo anterior, y según destaca Carlos Villán Durán, cada una de estas innovaciones posee una dudosa eficacia. En el primer caso, la mencionada disposición favorecerá a los Estados más ricos, los cuales pueden aportar con más recursos al programa de derechos humanos de la Organización. En cuanto a lo segundo, la eficacia práctica sería reducida, considerando que se deja a una mayoría cualificada de la Asamblea General, muy difícil de conseguir, la determinación que un Estado cometa o no violaciones sistemáticas a los derechos humanos. Respecto del tercer punto, Villán Durán sostiene que corresponde a una cláusula redundante, pues impone a los Estados miembros del Consejo las mismas obligaciones de comportamiento genéricas que ya poseen los Estados por el hecho de ser miembros de Naciones Unidas (Villán Durán, 2006: 2-3).

Por otro lado, la reducción del número de sus integrantes de 53 a 47 ha buscado aumentar su capacidad de actuar como foro y de llegar a decisiones consensuadas (New Zealand Ministry of Foreign Affairs and Trade, 2010: 28). Esta misma lógica se implementó para el nuevo Consejo Asesor, llamado a reemplazar a la Subcomisión de Derechos Humanos. Asimismo, la mayor relevancia del Consejo como un órgano directamente dependiente de la Asamblea General ha aumentado la capacidad de control de esta última. De esta forma, los miembros ya no serían elegidos por la mayoría de los 54 integrantes del ECOSOC, sino por la Asamblea General al reunir un mínimo de 96 votos a favor. 
A la Asamblea General también le corresponde confirmar o rechazar en última instancia, las decisiones del Consejo con respecto a la membresía de algunos países o de las declaraciones que emita, entre otros. Finalmente, el método de elección abierto e independiente, por países, aseguraría una competencia leal entre los candidatos y una toma de decisión transparente, mientras la rotación de sus integrantes buscaría reafirmar el proceso de democratización en el que se ha encaminado este organismo.

La Asamblea General, por el voto de los dos tercios de sus miembros presentes y votantes, puede suspender los derechos inherentes a formar parte del Consejo de Derechos Humanos a un Estado que cometa graves y sistemáticas violaciones a los derechos humanos. La suspensión de un miembro del Consejo de Derechos Humanos la resolverá la Asamblea General, sin la intervención del Consejo de Seguridad.

Asimismo, el Consejo de Derechos Humanos cuenta con un Comité Asesor como organismo subsidiario, que está compuesto por 18 expertos que se desempeñan a título personal, elegidos mediante el sistema de distribución geográfica. El Comité asesora al Consejo, mediante el aporte que los miembros otorgan con su experiencia, estudios e investigaciones.

\section{Estructura y relación con otras instituciones}

El Consejo de Derechos Humanos está formado por 47 miembros, electos de acuerdo con los grupos geográficos que representan. La distribución es la siguiente: 13 asientos para África, 13 para Asia y el Oriente Medio, ocho para Latinoamérica, seis para Europa del Este y siete países de Europa Occidental más Estados Unidos, Canadá, Australia y Nueva Zelanda.

Un cambio importante desde la entrada en vigencia del Consejo es el relativo a su ubicación en relación a los organismos de Naciones Unidas: durante su período de funcionamiento la Comisión de Derechos Humanos estaba bajo la jurisdicción del ECOSOC. Esta situación fue modificada, por lo que ahora el Consejo de Derechos Humanos tiene dependencia directa de la Asamblea General. Este nuevo nivel, le entrega al organismo una relevancia mayor y por tanto un grado de relación y de ingerencia superior en sus ámbitos de competencia. Aunque en esta línea, cabría considerar que el Consejo al tener la categoría de órgano subsidiario de la Asamblea General, carece de la visibilidad política de la categoría de órgano principal, que posee el Consejo de Seguridad o el ECOSOC.

Como parte del sistema de Naciones Unidas, el Consejo de Derechos Humanos se relaciona con otros organismos, tales como la Secretaría General, por cuanto de ella emanan las políticas, pero especialmente porque su tarea es brindar apoyo a todos los organismos de Naciones Unidas; el Alto Comisionado de Naciones Unidas para los refugiados (ACNUR), en razón de que su labor directa con los refugiados se relaciona con la 
agenda del Consejo en temas sobre discriminación, racismo y xenofobia; la Oficina del Alto Comisionado de Naciones Unidas para los Derechos Humanos (OHCHR); y la UNESCO, organismo que desarrolla estrategias de recomendación para la implementación de la temática de los derechos humanos en los currículos de escuelas, colegios y universidades de los países miembros, entre otros.

\section{El Examen Periódico Universal (EPU)}

Una de las reformas más relevantes del Consejo de Derechos Humanos se relaciona con los mecanismos de los que dispone esta nueva entidad. La anterior Comisión disponía de procedimientos de revisión temática o por países que se realizaban mediante relatores especiales, expertos independientes y grupos de trabajo. Estos mecanismos han sido conservados por el nuevo Consejo, que ahora además dispone de uno adicional de revisión periódica de todos los países, el denominado Examen Periódico Universal (EPU). Este nuevo mecanismo será evaluado luego de cinco años de su creación por la Asamblea General, cuando todos los países hayan presentado sus informes en dicho Examen en 2012.

Los informes periódicos universales son uno de los aspectos de cambio más importantes de este Consejo, por cuanto todos los países, sin exclusión, deben presentarlo y su preparación contempla la información suministrada por los distintos gobiernos sobre los avances, obstáculos y grados de comprensión de la temática de los derechos humanos. Según la resolución A/RES/60/251, el mecanismo garantiza la universalidad del examen y la igualdad de trato respecto de todos los Estados, basándose en un diálogo interactivo, con la plena participación del país de que se trate y teniendo en consideración sus necesidades en relación con el fomento de la capacidad.

Adicionalmente, el informe contiene dos partes importantes que son, por un lado la compilación de los informes de los comités realizados a partir de la información que suministran los Estados de manera obligatoria $^{6}$, y por otro, las perspectivas de la sociedad civil, lo que representa un importante avance, considerando que en el marco internacional sólo se contemplaba anteriormente la perspectiva de los Estados. En el caso de los países que no respeten los derechos humanos o sean responsables de violaciones graves y repetidas a éstos, se establece que podrán ser marginados del Consejo.

Otra de las novedades que involucra el EPU, se relaciona con el que todos los países firmantes deben presentarse una vez en un período de cuatro años para una revisión exhaustiva de su situación interna. Es así como por primera vez en la historia de Naciones Unidas, los países tradicionalmente poderosos -miembros de la Unión Europea, Estados Unidos, Rusia, Canadá, entre otros, son analizados y estudiados respecto de su situación interna en materia de derechos humanos. 
Tales innovaciones representan un avance histórico en el fortalecimiento del sistema de protección internacional de los derechos humanos, un avance en la democratización de Naciones Unidas, así como el impulso y fortalecimiento de la cooperación en el seno de la Organización en pos de dar una respuesta de manera eficaz a las violaciones y amenazas a los derechos humanos en el mundo entero.

Dentro de los principales criterios que se utilizan para la evaluación del EPU, se encuentran los siguientes:

- La ratificación de instrumentos internacionales de derechos humanos.

- El levantamiento de reservas a tratados de derechos humanos.

- La extensión de invitaciones abiertas y permanentes a los Procedimientos Especiales, para asegurar el desarrollo normativo temático. - La eliminación de la práctica de tortura y otros tratos o penas crueles, inhumanos o degradantes.

- Las acciones para erradicar todas las formas o manifestaciones de discriminación y violencia contra la mujer e igualdad de oportunidades.

- La protección de los derechos del niño y la niña, violencia, trata y responsabilidad penal juvenil.

- El asegurar el pleno ejercicio de la libertad de expresión y asociación.

- La abolición de la pena de muerte y/o establecimiento de una moratoria y;

- La creación de instituciones nacionales de derechos humanos.

El calendario del EPU se inició en 2008, con la Primera Sesión. En cada sesión son revisados 16 Estados. Cada país es revisado por una troika, compuesta por tres países elegidos previamente, que funcionan como relatores. El calendario contempla la revisión de todos los Estados en un período que va entre la $1^{\circ}$ sesión, en 2008 , hasta la $12^{\circ}$ sesión del año 2011.

En la línea de lo sostenido a lo largo de este trabajo, especialmente relevante ha sido la celebración del $9^{\circ}$ período de sesiones del EPU, celebrado entre el 1 y el 12 de noviembre de 2010, por cuanto, entre otros países, en ella fue revisada la situación de los derechos humanos en Estados Unidos. ${ }^{7}$ Este país presentó su primer Informe Nacional en un escenario de gran expectación. ${ }^{8}$ El Informe redactado por el Departamento de Estado destacaba los logros que ha tenido Washington para asegurar que todos los estadounidenses sean iguales ante la ley. No obstante, se reconoce que queda mucho por hacer. Entre las principales recomendaciones que recibió el país se destacan las siguientes: Cierre de la prisión de Guantánamo; se demandó la abolición de la pena capital vigente en algunos Estados del país; se manifestó preocupación por la agenda migratoria; y se recomendó que Estados Unidos prohíba el uso de estereotipos o perfiles raciales en la aplicación de la ley migratoria. 
Asimismo, la mayoría de las delegaciones instaron a Estados Unidos a ratificar tratados internacionales, entre los que destacan el Estatuto de Roma, la Convención sobre la eliminación de todas las formas de discriminación contra la mujer y la Convención sobre los derechos de las personas con discapacidad. Como respuesta a las recomendaciones, Estados Unidos anunció que éstas serían analizadas y que pronto éste país otorgaría una respuesta a las consideraciones expresadas sobre el particular.

De ello se desprende que, en gran parte, la fortaleza y legitimidad del futuro órgano dependerá de la exitosa implementación del nuevo mecanismo de revisión periódica, así como de la voluntad política de los países. De todos modos, el mismo hecho que Estados Unidos se haya sometido al procedimiento, demuestra el importante grado de democratización e imparcialidad que ha ganado la comunidad internacional con el nuevo organismo.

\section{Agenda del Consejo de Derechos Humanos}

Los temas generales de la agenda del Consejo de Derechos Humanos fueron concebidos durante su creación y se vienen precisando durante las sesiones de trabajo. Los siguientes son los puntos sustantivos de la Agenda del Consejo de Derechos Humanos: Promoción y Protección de los Derechos Humanos; Derechos económicos, sociales y culturales; Derechos civiles y políticos; Derechos de las personas y grupos específicos e individuales; Temáticas transversales de derechos humanos; Situaciones de derechos humanos que requieren de la atención del Consejo; Derecho al desarrollo; Cuerpos y mecanismos de los derechos humanos, el EPU; Situación de los derechos humanos en Palestina y los otros territorios árabes ocupados; Conferencias y cumbres de las Naciones Unidas relativas a los Derechos Humanos, Racismo, discriminación racial, xenofobia y formas relacionadas de intolerancia, seguimiento e implementación de la Declaración y el Programa de Acción de Durban; la Declaración y el Programa de Acción de Viena; y Seguimiento de las decisiones del Consejo de Derechos Humanos.

El Consejo funciona como una serie de interrelaciones de grupos encabezados por un país líder que, en un sentido positivo (si impulsa) o negativo (si frena), es capaz, gracias a los recursos de poder de que dispone, de agrupar a su alrededor a los países menores. Los países que lideran estas iniciativas no corresponden necesariamente a los miembros permanentes del Consejo de Seguridad de Naciones Unidas, sino que dependen del tema específico que eventualmente se trate. En ocasiones, ciertos países con liderazgo en torno a determinados temas son capaces de encabezar iniciativas a nivel regional.

Dentro de los países que destacan en el marco de los grupos regionales, se puede identificar a Egipto y Sudáfrica en los Estados africanos; Brasil y México en América Latina; China, India Japón y Arabia Saudita en 
los Estados asiáticos; Francia y Gran Bretaña en el grupo de la Unión Europea y Otros; mientras que en el grupo de los países del Este de Europa, destaca especialmente Rusia. Estos países lideran y ejercen algún grado de poder en sus respectivos grupos, ya sea para impulsar iniciativas como para protegerse de ellas.

Algunos Estados actúan bajo una lógica pragmática, mediante el uso indiscriminado de debates que dilatan la adopción de consensos. Por otra parte, Estados fuertemente cuestionados por las violaciones a los derechos humanos, como es el caso de China, por cierto que prefieren pertenecer al Consejo, tal vez no con la convicción de cambiar sus conductas, sino como medio de resguardo en la medida que pueden ejercer cuotas de poder dentro del mismo.

Por otra parte, un actor importante que resalta es Estados Unidos, país que a pesar de haber votado en contra de la creación del Consejo durante la presidencia de George W. Bush-, se ha comprometido formalmente en apoyar su quehacer. Lo anterior tiene importantes consecuencias, si se considera que las autoridades estadounidenses han expresado en variadas ocasiones su postura contraria, no sólo a las instituciones internacionales que interfieran con sus intereses nacionales, sino especialmente con Naciones Unidas (véase Remiro Brotóns, 2003). La crítica de Estados Unidos respecto de los reales aportes de la Organización no sólo se relaciona con el aporte económico de este país al presupuesto de Naciones Unidas, sino que va más allá, considerando que muchas de las resoluciones que adopta son contrarias a sus intereses nacionales, transformándose luego en principios de derecho internacional. Sin embargo, la administración Obama ha otorgado señales claras de apoyo a la labor del Consejo, así como a las tendencias generales de la política multilateral global.

Por último, cabe señalar que el Consejo aborda diversos temas de derechos humanos, que son impulsados por diferentes Grupos de Trabajo, a saber: Grupo de Trabajo sobre el Derecho al Desarrollo; Grupo de Trabajo Intergubernamental sobre la Aplicación Efectiva sobre la Declaración y el Programa de Acción de Durban; Grupo de Trabajo de Expertos sobre las personas de Ascendencia Africana; Comité Ad Hoc sobre la Elaboración de Estándares complementarios y el Proceso Abierto del Grupo de Trabajo sobre un Protocolo Facultativo a la Convención sobre los Derechos del Niño.

\section{Materias prioritarias que son motivo de reflexión y búsqueda de consensos}

Entre las variadas materias que se han analizado en el Consejo de Derechos Humanos, una de las que resalta con más fuerza se refiere a la participación efectiva de las minorías en la vida social, económica y política de los Estados. A este respecto, cabe sostener que la exclusión de las minorías sigue preocupando a la comunidad internacional. La participa- 
ción de estos grupos en la vida económica, particularmente en tiempos de crisis, corresponde a una condición esencial para mejorar los niveles de inclusión. Cuando las minorías tienen la oportunidad de contribuir con su trabajo, creatividad y habilidades empresariales, las sociedades se benefician importantemente. Asimismo, se debe plantear que la plena participación de las minorías debe ser enfocada desde una perspectiva de derecho, como un componente clave en la construcción de sociedades equitativas y estables.

Otro tema que destaca es la necesidad de incrementar la eficiencia del Consejo de Derechos Humanos. Sobre el particular, hay que subrayar que la efectividad del Consejo no sólo debe tratarse bajo la perspectiva de un ejercicio procesal. Por tal motivo, se requiere ampliar los plazos para las consultas y aumentar la disponibilidad de los recursos en razón de las implicancias financieras que conlleva la implementación de algunas resoluciones.

Asimismo, se necesita orientar la labor del Consejo de Derechos Humanos hacia el aumento del impacto de su trabajo a nivel nacional. En este sentido, cabe subrayar que resulta indispensable que la acción del Consejo genere mejoras en los estándares nacionales, mediante el impulso de instrumentos legislativos y programas concretos. Sobre este aspecto, es dable recalcar que la labor debiera contemplar los aspectos normativos, culturales y la voluntad política de los Estados. Se hace necesario también aumentar la cooperación técnica con el fin de mejorar las capacidades de los países.

Por último, también resalta como tema el mejoramiento de la acción del Consejo en países específicos, en situaciones de emergencia. En este punto, se debe señalar que, a pesar de que el EPU ha sido una contribución enorme a la reducción de la selectividad y la politización del tema de los derechos humanos en el mundo, la polarización de la antigua Comisión aún subsiste. Este tema requiere de un enfoque que integre la cooperación y el diálogo constructivo con los países involucrados, así como de la utilización de las herramientas con que cuenta el Consejo para hacer frente a situaciones de emergencia, ante la necesidad de incrementar la cooperación técnica y el fomento de capacidades.

\section{Primer proceso de Revisión del Consejo de Derechos Humanos}

Conforme a lo establecido en la resolución A/RES/60/251, que estableció la creación del Consejo de Derechos Humanos, se inició en Ginebra, a principios de 2010, y en Nueva York, en diciembre del mismo año, el proceso de revisión del organismo referido al estatus del mismo. La pretensión de una gran cantidad de Estados era elevar la categoría del Consejo desde ser un órgano subsidiario de la Asamblea 
General a ser un órgano principal - autónomo- del sistema de Naciones Unidas, para lo cual se requiere, por cierto, una modificación de la Carta de Naciones Unidas.

Este proceso estuvo liderado por dos co-facilitadores, los Representantes Permanentes ante Naciones Unidas de Liechtenstein y Marruecos. En la ocasión se conformaron varios grupos denominados likeminded, los cuales agruparon a Estados que comparten intereses y puntos de vista respecto del futuro del organismo. Por ejemplo, los grupos integrados por países árabes, africanos y la Conferencia Islámica tuvieron una participación importante con una visión conservadora respecto de lo que debiera ser el funcionamiento del Consejo, quitándole posibilidades de desarrollo mediante el otorgamiento de mayores atribuciones. Junto a estos grupos, compartieron visiones conservadoras un grupo trans-regional liderado por la Federación Rusa e integrado por Cuba, Bolivia, Nicaragua -entre otros-, que presentaron posiciones alejadas del espíritu inicial de la revisión. Se rechazó de plano la propuesta de realizar una presentación pública de los compromisos de los Estados, que incluyera opiniones de la sociedad civil. Esta propuesta no intentaba establecer nuevos criterios respecto de la membrecía al organismo, sino operacionalizar el párrafo operativo 8 de la resolución 60/251.

Respecto de los compromisos que los Estados miembros manifiesten respecto de la promoción y protección de los derechos humanos, indicados en el párrafo 8 de la mencionada resolución, cabe subrayar que la Asamblea General, por mayoría de dos tercios, suspendió a Libia su membrecía en el Consejo en marzo de 2011, mediante la resolución A/RES/65/265, por violaciones graves y sistemáticas a los derechos humanos.

Este proceso de revisión del Consejo, establecido en el párrafo operativo 16 de la resolución A/RES/60/251, no logró consensuar un cambio en el estatus del organismo, sin embargo, se dejó abierta la posibilidad de volver a plantear el tema de su jerarquía.

El segundo ciclo para efectuar el EPU se llevará a cabo entre 2012 y 2016. Una vez se vuelvan a revisar todos los Estados de Naciones Unidas. Los países de corte más liberal, entre los que se cuentan los integrantes de la Unión Europea, y algunos latinoamericanos, esperan poder realizar una segunda revisión con la esperanza de perfeccionar este organismo y elevar su estatus al de órgano principal de Naciones Unidas. De acuerdo a lo establecido en la Cumbre de 2005, que generó el proceso de establecimiento del Consejo de Derechos Humanos, se definieron tres pilares fundamentales donde descansa el bienestar general del mundo, la paz y seguridad internacionales, el desarrollo y los derechos humanos. Este último, requiere de un órgano especializado que vele por el perfeccionamiento de las normas que regulan y resguardan la convivencia humana. 


\section{Conclusiones}

La creación del Consejo de Derechos Humanos representa indudablemente un cambio en el énfasis que quiere otorgar Naciones Unidas al tema de los derechos humanos. En las expectativas de sus impulsores, se espera que el nuevo organismo contribuya a la resolución de los problemas de ineficacia y politización que afectaron a su antecesora. Adicionalmente, con ello se busca dar un enfoque de universalidad y transversalidad al tema de los derechos humanos.

La paulatina consolidación de la estructura de los informes del EPU ha sido fundamental para el fortalecimiento del Consejo y su afirmación como órgano de referencia en el ámbito de los derechos humanos. Desde el punto de vista de la realización de los informes y de las recomendaciones que han surgido, resulta notable el avance y el sometimiento de los Estados ante las recomendaciones que se les han realizado. Un caso paradigmático ha sido el de Estados Unidos, que presentó su Informe el año 2010. El apoyo estadounidense resulta fundamental para cualquier iniciativa que se intente llevar a cabo en el seno de la comunidad internacional. Sin duda, la administración Obama ha tenido una importante cuota de responsabilidad a este respecto.

Por otro lado, el Consejo -dentro del sistema de Naciones Unidas- está marcando una pauta que, puesta en perspectiva, puede resultar en un antecedente relevante para toda la Organización. Mediante este Consejo se ha instalado con fuerza un criterio universal geográfico más representativo y democrático, así como integrador de los países menos adelantados, que seguramente se considerará en la reestructuración de Naciones Unidas.

Asimismo, la efectiva participación de la sociedad civil en la elaboración de los informes, se reconoce como un gran logro, pues permitirá formalizar la visión de una importante parte de la sociedad en un escenario global al que se han agregado con fuerza nuevos actores internacionales y transnacionales. También es una tarea en la que se debe avanzar mediante la educación y la información de forma trasversal a toda la sociedad, pues corresponde a una parte crucial del informe.

Pese a lo anterior, todavía numerosos países ven con cierto recelo al Consejo de Derechos Humanos, al que perciben como un instrumento que valida una mirada occidental en estas materias. Al mismo tiempo, se visualiza que aún persisten concepciones tradicionales respecto de la soberanía de los Estados, en particular, en aquellos países con menores niveles de apertura, como es el caso de algunos países del medio y lejano Oriente. Los sucesos que se experimentan al interior de estos países son concebidos en éstos como dominio exclusivo de las autoridades nacionales, donde el rol de la sociedad es limitado y muchas veces controlado. Por lo anterior, estos países son reticentes a la incorporación de las ONGs en los debates y opiniones al interior del organismo, debido al recelo que les producen y el 
prejuicio respecto de su concepción ideológica y sus poco claras fuentes de financiamiento.

En cuanto a los resultados de la primera revisión del Consejo de Derechos Humanos, se logró mantener la fórmula consagrada en la resolución que lo creó, la que otorga la oportunidad de volver a plantear el tema del estatus de la entidad en una segunda revisión, la cual se efectuará en 2016. Lo anterior, tomando en cuenta la férrea oposición que mantuvieron los países más conservadores, entre los que se encuentran Estados africanos, árabes y algunos latinoamericanos.

El desafío futuro del Consejo es entrar en una negociación substantiva sobre el avance en los estándares internacionales, evitando el retroceso y estimulando respuestas que mejoren la efectividad de éste, con el fin de reforzar su propia legitimidad y la del sistema de Naciones Unidas como un todo. 


\section{Notas}

${ }^{*}$ Este trabajo es de exclusiva responsabilidad de los autores y no representa la opinión del Ministerio de Relaciones Exteriores de Chile. Un avance de esta investigación se encuentra en Acevedo y Riquelme (2011).

${ }^{1}$ Según se establece en dicho documento, las negociaciones deben conducirse de buena fe; los Estados deben tener en cuenta la importancia de participar de modo apropiado; el propósito y objeto de todas las negociaciones debe ser compatible con los principios y normas del derecho internacional y de la Carta de Naciones Unidas; los Estados deben adherir a un marco acordado mutuamente y esforzarse por mantener una atmósfera constructiva y abstenerse de toda conducta que pueda afectar las negociaciones y sus progresos; los Estados deben facilitar la búsqueda o conclusión de sus negociaciones manteniéndose concentrados en los objetivos principales y; los Estados deben emplear sus mayores esfuerzos para continuar trabajando por una solución justa y aceptable.

${ }^{2}$ Generalmente estas peticiones se efectúan cuando algún Estado se encuentra ante presiones internas o de normas nacionales, que impiden aceptar algunas acciones propuestas. Por ello, los negociadores piden una mayor flexibilidad o el intercambio de apoyos en otras cuestiones.

${ }^{3}$ En el Considerando 3 de la Declaración del Milenio (A/RES/55/2, del 13 de sep. de 2000), se deja constancia de lo siguiente: "Reafirmamos nuestra adhesión a los propósitos y principios de la Carta de las Naciones Unidas, que han demostrado ser intemporales y universales. A decir verdad, su pertinencia y su capacidad como fuente de inspiración han ido en aumento conforme se han multiplicado los vínculos y se ha consolidado la interdependencia entre las naciones y los pueblos”.

${ }^{4}$ El texto que recoge las declaraciones de los países (A/60/PV.72), se encuentra disponible en URL: http://daccess-dds-ny.un.org/doc/UNDOC/GEN/N06/272/63/PDF/N0627263. pdf?OpenElement Recuperado en julio de 2011.

${ }^{5}$ Resolución A/RES/60/251 de la Asamblea General, Párrafo Operativo 10.

${ }^{6}$ Dichos informes son compilados por la oficina del Alto Comisionado para los Derechos Humanos.

${ }^{7}$ Los otros países revisados en la ocasión fueron Liberia, Malawi, Mongolia, Panamá, Maldivas, Andorra, Bulgaria, Honduras, Islas Marshall, Croacia, Jamaica, Libia, Micronesia, El Líbano y Mauritania.

${ }^{8}$ Estados Unidos tuvo como relatores a Camerún, Francia y Japón. Los Informes de Naciones Unidas y de las ONG sobre la situación de los derechos humanos en Estados Unidos están disponibles en URL: http://www.ohchr.org/ENHRBodies/UPR\%5CPAGES\% 5CUSSession9.aspx Recuperado en diciembre de 2010. 


\section{Bibliografía}

Acevedo, Irene y Jorge Riquelme (2011), "Los derechos humanos y los nuevos mecanismos en Naciones Unidas”. En Iberoamérica, N².

Figueroa, Uldaricio (2010), Organismos Internacionales, Tomo I. Ril Editores, Santiago de Chile.

Morandé, José (2004), “Notas y alcances sobre el Estado-nación en la política mundial del presente: una reflexión desde las Relaciones Internacionales”. En Estudios Internacionales, № 145.

Naciones Unidas (2000), ABC de las Naciones Unidas. Departamento de Información Pública de las Naciones Unidas, Nueva York.

New Zealand Ministry of Foreign Affairs and Trade (2010), United Nations Handbook 2009-2010. Forty-seventh edition, Wellington.

North, Douglass (1990), Institutions, Institutional Change and Economic Performance. Cambridge University Press, New York.

Remiro Brotóns, Antonio (2003), “ ¿Nuevo orden o derecho internacional?”. En Claves de Razón Práctica, Nº 132.

Villán Durán, Carlos (2006), “Luces y sombras del nuevo Consejo de Derechos Humanos de las Naciones Unidas”. En Eikasia. Revista de Filosofía, $\mathrm{N}^{\circ} 4$.

Zalaquett, José (2010), "Los fundamentos de los derechos humanos, su evolución y los desafíos actuales”. En Cuadernos de Difusión (Centro de Estudios Internacionales CEIUC), $\mathrm{N}^{\circ} 6$, año 3.

Recibido: 14.01.2011

Aceptado: 14.09.2011 